\title{
Mentoring during the COVID-19 pandemic: a perspective from graduate students in biomedical science studies in India
}

\author{
Purna Bapat $^{1}$ - Shilpa Bhat ${ }^{2}$. Camellia Chakraborty ${ }^{3}$. Narendra Chirmule ${ }^{4}\left(\mathbb{0} \cdot\right.$ Rajashree Kadam $^{1} \cdot$ Sukesh Kashyap $^{3}$. \\ Shalaka Masurkar ${ }^{1} \cdot$ Raikamal Paul $^{1} \cdot$ Dipti Rao $^{5} \cdot$ Reshma Reddy $^{1} \cdot \operatorname{Trupti}$ Togar $^{1}$
}

Received: 25 January 2021 / Accepted: 22 February 2021 / Published online: 2 June 2021

(c) Indian National Science Academy 2021

\begin{abstract}
Mentorship transforms careers. Yet, a lack of awareness of the need for a mentor is ironically, a pandemic. The pandemic has brought a host of challenges for the academic researcher. As bench work has come to a standstill, there is not just reduced productivity, but a significant increase in fear, anxiety, and depression amongst students. Our survey of - 150 graduate students of Life Sciences courses confirmed the anecdotal observation that $>75 \%$ did not have a mentor other than their academic guide. Over the past six months, we have been exploring the requirements of mentee-mentor relationships during both normal times and during the COVID-19 pandemic. Written primarily from the mentee perspective, we have described the (i) criteria and roles of an ideal mentor and mentee, (ii) the problems faced by the mentee, and (iii) the solutions to address these challenges. We have described the experience of 10 mentees with a mentor during these challenging times, in which we discussed scientific, psychological, and practical support for each other. We provide some recommendations based on previous work in mentor-mentee interactions and explore the critical nature of this delicate relationship for a successful career.
\end{abstract}

Keywords Mentor $\cdot$ Empathy $\cdot$ COVID-19 $\cdot$ Scientific $\cdot$ Emotional $\cdot$ Support $\cdot$ Networking

\section{Introduction}

The COVID-19 pandemic has disrupted normal physical interactions in unprecedented ways (Gotian 2020). Public lockdowns have resulted in significant mental instability; the need for kindness and compassion is more than ever, both in the workplace and at home. In the highly structured world of academia, work has been severely disrupted, leading to uncertainty of how to move forward, a lack of understanding about responding to situations caused by the pandemic, and the lack of a support system.

This article is part of the Special Issue: Indian National Young Academy of Science (INYAS).

Narendra Chirmule

chirmule@gmail.com

1 Advanced Center for Treatment, Education and Research in Cancer (ACTREC), Kharghar, Nav-Mumbai, India

2 Mu-Sigma, Bangalore, India

3 IIT, Gandhinagar, Gandhinagar, India

4 SymphonyTech Biologics, Pune, India

5 Art-X Company, Bangalore, India
A recent article in PNAS by Ma et al. (2020) analyzed data on 40,000 scientists, who published 1,167,517 papers in biomedical journals between 1960 to 2017, to investigate the relationship between mentors and protégé. The study revealed three major findings: (i) Mentorship predicts the success of the protégé, (ii) Protégés typically pioneer work in their field, and (iii) Surprisingly, mentees succeed by studying original topics different from that of their mentors. Several such studies on mentor-mentee relationships have emphasized the critical role this interaction has on the success of the mentee.

Studies on mentor-mentee interactions have also established that having a mentor translates to exponentially successful careers (Ma et al. 2020; Dam 2020; Shanmukhappa 2020). The roles and responsibilities of both mentors and mentees have been described extensively (Eby and Robertson 2020; Tyler 2018). Despite numerous books, scientific publications, and TED talks (Ortiz 2019; Nelson 2019), the requirement of mentors during training in graduate schools is routinely undervalued. This systemic lack of resource for the graduate student can be attributed to the dominant role of the academic advisor, often mistaken to be that of a mentor. Our survey confirms this misunderstanding and provides potential solutions for enabling a successful mentorship. 


\section{Problem definition}

The academic guide is a central figure in the life of a graduate student. They take on the roles of professor, scientific consultant, and head of the research unit amongst others. However, the academic guide is rarely explicitly charged with the student's wellbeing, emotional and mental health, motivation, and personal development. In this most challenging of professional pursuits, the subtle needs of the graduate student largely go unseen and consequently have long-lasting effects on the individual.

\section{The mentor-mentee relationship}

\section{Roles of a mentor}

Effective mentors embody the following characteristics and actions: they (i) establish trust and allow mistakes; (ii) are open to reciprocal feedback; (iii) learn from the mentee; (iv) set realistic goals and expectations; (v) demonstrate flexibility; (vi) become a partner in problem-solving; (vii) work on common projects and tasks; (viii) are aware of the limitations of mentoring; (ix) are genuinely concerned about the mentee; (x) aid in overall personality development of the mentee.

\section{Role of a mentee}

Mentoring is a two way process with equal contribution from both mentor and mentee. A mentee therefore must: (i) trust the mentor and their approach or method of mentoring; (ii) take constructive criticism positively and work with it; (iii) work to develop realistic plans; (iv) invest in the process wholeheartedly; (v) avoid having unrealistic expectations from the mentor.

\section{Comparison between a mentor and academic guide}

To a graduate student, the academic guide is the primary force that aids the progress of the student's research and offers scientific input. This relationship includes a sense of hierarchy, an authoritative distance, and limited scope. However, the progress of a mentee in this relationship is limited to academic pursuits, which often overshadows the need for personal and professional development. As a more equitable association the mentoring relationship addresses aspects beyond research and lab work. In the case of a PhD where researchers develop a narrow focus, a mentor can guide the student to think creatively. Unlike in a relationship with an academic guide, a student can talk openly to a mentor about emotional distress.

\section{Challenges during a graduate program}

The pursuit of the most rigorous form of academic study, the $\mathrm{PhD}$, has been made even more challenging in the post-pandemic world. The potential challenges in the path to a doctorate include changing goals, uncertain time commitment, poor work-life balance, financial insecurity, and uncertainty of career prospects. In addition to the stress of academic work, the pressure of publishing, harassment, discrimination and biases, failures of experiments, unmet expectations (Woolston 2019) and limited funding, all take a heavy toll on physical and mental health. Graduate students often experience burnout and fatigue, and in extreme cases, suffer from anxiety and depression (Koutsimani et al. 2019). In India in particular, the stresses of a doctorate often cannot be discussed with families that are unfamiliar with the research environment. Here, since March 2020, a large number of graduate research students living on campus were asked to return to homes before the national lockdown was instituted. In the field of life sciences, as lab work is impossible to replicate outside of scientific laboratories and forms a core part of research work, the lack of access to research resources has led to massive disruptions in schedules and research work. The world of academia further stands to lose a large number of students and researchers who come from vulnerable financial backgrounds and need to support families.

\section{Proposed solutions and a way forward}

Signs of anxiety and depression among graduate students have doubled during the pandemic (Woolston 2020). The uncertainty of the future and a sense of hopelessness has contributed to the angst. In order to support the mental health of the students, we have listed several potential solutions that can be undertaken: (i) acknowledge the crisis and recognize students' challenges; (ii) actively communicate openly and transparently and create for a for discussion between students and institutions; (iii) review and revise research plans and consider alternatives like in silico studies; (iv) provide opportunities for orthogonal activities based on student interest; (v) encourage upskilling during 'downtime'; (vi) offer counselling; (vii) use DISC (2021) or Meyers-Briggs tools (2019) to perform assessments for inclusive solutions; (viii) bolster emotional, physical, and spiritual health.

Limitations and uncertainties to implement the recommendations: 
- Each institute may not have resources allocated for mentorship or counsel the mentee.

- Professionally trained personnel may not be available for helping the students.

- Many projects may not have the option of in silico alternatives.

- There may be no internal process to monitor the emotional and mental health of the students.

- There may be no external assessment of the internal mentoring process, and criteria suitable for evaluation.

- There may be lack of funding for an overall mentoring program.

- A process to plan for dealing with crisis situations (e.g., pandemic) may not be in place.

- The survey was not random since it was conducted in only a few institutions.

- The survey may have a bias to interpret this data set for the mentoring process.

The understanding of the limitations may provide opportunities to create an environment and infrastructure for an effective mentorship program for graduate students.

\section{Survey}

While the authors have shared their own experiences and understanding of the mentoring process, it was important to gain an understanding of the wider graduate student perspective on the topic. The survey sought to examine graduate students' approach to mentorship, and their expectations from this relationship. We received a total of 210 responses through the authors' peer networks, colleagues, friends, and acquaintances. The dissemination was not random and therefore the authors do not claim the data is random and representative of the population. Of the 210 respondents, only responses from students based in India and working in the life sciences field were considered, bringing the total responses examined to 150 . Broadly, respondents were classified into two groups: (i) students who had a mentor as well as an academic guide, or Group A; (ii) students who only had an academic guide, whom they consider as mentor or Group B.

\section{Analysis of the survey}

We received a total of 210 responses in the survey. Of these most responses were from India (175 of 210). Of the Indian respondents, most were from life-science streams (150 of 175) and hence we only used such students in analysis to enable comparison in a specific population. Fields such as biochemistry, bioengineering were grouped into life sciences. Therefore, our final analysis cohort consisted of respondents from India studying in life sciences domain. Significance testing was done on our hypotheses using p-values with Cramer's V for effect size. However, since the sample collection was distributed by the authors to their networks, and not random sampling, our hypotheses was limited to the 150 responses. Mean ratings were used to draw most conclusions.

\section{Results: insights into the expectations of PhD students from an academic guide vs. a mentor}

1. An academic guide is expected to give scientific input as a primary role.

2. An academic guide is expected to help with financial assistance primarily.

3. A mentor is expected to provide emotional support.

4. Academic Guides and Mentors both should provide operational support.

The survey responses indicate that students in Group A who had both an academic guide and mentor expected each person to take on different roles: for mentors to provide emotional support and for academic guides to focus on research support and financial assistance. Both groups however, expected both academic guides and mentors to be approachable, provide time for discussion, offer operational support, and provide networking support. Mentors were consistently rated higher than academic guides in areas of 'soft skills' including empathetic listening, emotional support. Importantly, respondents who had both mentors and academic guides rated themselves less affected than those with only academic guides.

\section{Examining the understanding of the concept of mentoring amongst respondents}

The pattern of responses from students with and without mentors pointed to key areas of understanding of the concept of mentorship:

- In the absence of a mentorship experience, students have greater expectations of a mentor. This may point to an unmet need for more support from a guide.

- Students without the experience of a mentor do not expect emotional support from a mentor.

- Academic guides are expected to provide emotional support regardless of whether a mentor is in the picture. This may point to a need for academic guides to consciously focus on providing such support. 


\section{Conclusion}

The past 6 months have been extremely useful in evaluating the mentoring processes for graduate students. The observations in this report are a summary of the 10 authors of this paper, and some information from 200 participants in the survey. We have provided a list of potential solutions to the challenges that graduate students are experiencing. These notes should be utilised and applied, as required, at both the individual and institutional levels. The report also underscores the requirement of a larger effort for developing an ecosystem for mentoring support that will provide mentees with resources after the pandemic, in the "new normal".

Supplementary Information The online version contains supplementary material available at https://doi.org/10.1007/s43538-021-00002-z.

\section{References}

DISC Assessment. https://www.everythingdisc.com/. Accessed 15 Feb 2021

Eby, L.T., Robertson, M.M.: The psychology of workplace mentoring relationships. Ann. Rev. Org. Psychol. Org. Behav. 7(1), 75-100 (2020)
Gotian, R.: Mentoring during the COVID-19 pandemic. Nature (2020). https://doi.org/10.1038/d41586-020-01028-x

Koutsimani, P., Montgomery, A., Georganta, K.: The relationship between burnout, depression, and anxiety: a systematic review and meta-analysis. Front. Psychol. 10, 284 (2019)

Ma, Y., Mukherjee, S., Uzzi, B.: Mentorship and protege success in STEM fields. Proc. Natl. Acad. Sci USA 117(25), 14077-14083 (2020)

Meyers Briggs Type Indicator https://www.myersbriggs.org/my-mbtipersonality-type/mbti-basics/ (2019). Accessed 15 Feb 2021

Nelson, R.: The power of mentoring. TED Talk. (2019). https://www. ted.com/talks/reggie_nelson_the_power_of_mentoring.

Ortiz, K.: How to be a great mentor. TED Talk. (2019). https://www. ted.com/talks/kenneth_ortiz_how_to_be_a_great_mentor.

Shanmukhappa, S.C., et al.: Motivators and barriers to research among doctors in the Indian medical scenario: a cross-sectional study from Karnataka, India. J. Fam. Med. Prim. Care 9(8), 4053-4061 (2020)

Tyler, K.: Mentoring that matters. HRMagazine, pp. 28-35 (2018)

van Dam, L., et al.: Youth initiated mentoring: a meta-analytic study of a hybrid approach to youth mentoring. J. Youth Adolesc. (2020). https://doi.org/10.1007/s10964-020-01336-5

Woolston, C.: PhDs: the tortuous truth. Nature 575(7782), 403-406 (2019)

Woolston, C.: Pandemic and panic for US graduate students. Nature $\mathbf{5 8 5}, 147-148(2020)$ 